\title{
The Néel Magnetic Relaxation Dynamics in Nanoparticle Systems — Phenomenological Approach versus Stochastic Approach
}

\author{
M. OsACI* \\ University "Politehnica" of Timisoara, Engineering Faculty of Hunedoara, \\ Department of Electrical Engineering and Industrial IT, Revolutiei no. 5, Hunedoara, Romania \\ (Received November 11, 2013; revised version January 30, 2016; in final form March 2, 2016)
}

\begin{abstract}
In nanomagnetism, the studies of magnetic nanoparticle systems are of particular interest from both experimental and theoretical points of view. Experimentally, the measurements made on such a system are hard to interpret. It is very difficult to distinguish the effect of the magnetic dipole interactions from the effects of size distribution or effective magnetic anisotropy constants. In this respect, the simulation models can help. This paper presents a study comparing the two conventional approaches, using simulation models for the magnetic relaxation dynamics of nanoparticle systems, i.e. a phenomenological Ising-type approach, on two levels, and a stochastic approach. The paper also shows a way of using these approaches in creating a model to simulate the Néel magnetic relaxation time for aligned magnetic nanoparticle systems.
\end{abstract}

DOI: 10.12693/APhysPolA.129.323

PACS/topics: 72.15.Lh, 75.50.Tt, 78.20.Bh

\section{Introduction}

The nanoparticle magnetic system $[1,2]$ is expected to become one of the most versatile tools in biotechnology, biomedical, material science, and engineering [35], having a number of important applications, such as magnetic fluid hyperthermia for cancer treatment [6-9], magnetic resonance imaging, drug targeting, biomarkers, biosensors and separation of biomolecules [2, 10, 11], and ultra-high density magnetic data storage devices [12]. Most applications are using external high frequency alternating magnetic field. The nanoparticles are randomly distributed in a given volume, their sizes and effective anisotropy constants have distributions and, in general, we may take into account the dipolar magnetic interactions. In these systems, the dynamics of nanoparticles is characterized by the Néel relaxation time and Brownian relaxation time, given that the nanoparticles are in suspension in a liquid base. Due to the complexity of these systems, the studies are using modelling and numerical simulations which require certain methods of calculation. Therefore, the magnetic relaxation dynamics in nanoparticle systems represents a topic of interest in the recent decades. There are a lot of models with many approximations, trying to provide solutions, but which lead to more or less controversial results.

The string of models started with the work of Néel [13], for nanoparticles that do not interact, and continued with Brown's concerns [14] and attempts to find a solution to the Fokker-Planck equation for the orientation probability density of the nanoparticle magnetic moment, as a starting point for the relaxation time calculation.

*e-mail: mihaela.osaci@upt.ro
The first attempt to introduce the interactions into the Néel-Brown model was made by Shtrikmann and Wohlfarth who, by using a mean field approximation, predicted a Vogel-Fulcher law for the relaxation time in the weak interaction limit [15]. A more general approach was developed by Dormann et al. [16], and Hansen and Mørup [17]. Both models took into account the dipole-dipole interaction, but achieved conflicting results. To these models, we can add many studies carried out using the Monte Carlo stochastic methods [18-20], or the numerical solution of the Landau-Lifshitz-Gilbert stochastic differential equation (LLG equation) [21], or rigorous analytical models $[22,23]$. Unfortunately, they could offer only approximate solutions, or solutions for weak magnetic dipole interactions. In many of these models, we can find stochastic or phenomenological approaches. For example, one of the most updated models, based on the KMC method (kinetic Monte Carlo method), uses a stochastic approach [20]. Thus, for effective local magnetic fields of particles whose density is lower than a critical value, there are two equilibrium nanoparticle states, "+" and "-", and the probability of a nanoparticle to switch between these states is $P_{i}=1-\exp \left(-t / \tau_{i}\right)$. The relaxation time constant $\tau_{i}$ is a reciprocal sum of the transition rates $\tau_{i}^{+}$and $\tau_{i}^{-}$, dependent on the energy barriers $\Delta E_{i}^{ \pm}$, seen from the "+" and "_" states via the standard Néel-Arrhenius law: $\tau_{i}^{ \pm}=\tau_{0} \exp \left(\Delta E_{i}^{ \pm} / k_{\mathrm{B}} T\right)$, where $k_{\mathrm{B}}$ is the Boltzmann constant, and $T$ is the temperature [20].

Further, we could find an interesting study comparing the two approaches found in various models for the magnetic relaxation dynamics in nanoparticle systems, i.e. a phenomenological approach of the Ising type, on two levels, and a stochastic approach. These approaches can be considered in aligned magnetic nanoparticle systems. These approaches may take into account distributions 
of sizes and effective magnetic anisotropy constants, geometric distribution of the nanoparticles in a given volume and dipolar magnetic interactions, characteristics specific to the real systems. Thus, we can work locally, at the level of particle, with mediation for the physical quantities of interest.

\section{Local magnetic field}

\section{in case of aligned magnetic nanoparticle system}

In case of oriented nanoparticles, if we take into account the nanoparticle interactions, we can consider that the local magnetic field over the given $i$ nanoparticle is made of two contributions: the external magnetic field acting along the easy magnetisation direction, $O z$, and the projection along the external field of the dipolar magnetic field created on the $i$ nanoparticle due to the dipolar magnetic interactions of the nanoparticles. The other projections may be neglected, because they are small compared with the projections in the direction of the $O z$ axis

$$
\begin{aligned}
& H_{i}=H+H_{\mathrm{d} z, i}, \\
& \boldsymbol{H}_{\mathrm{d}, i}=\frac{1}{4 \pi} \sum_{j, j \neq i} \frac{\mu_{j}}{r_{i j}^{3}}\left[3 \widehat{r}_{i j} \cdot\left(\widehat{\mu}_{j} \cdot \widehat{r}_{i j}\right)-\widehat{\mu}_{j}\right],
\end{aligned}
$$

where $r_{i j}$ is the distance between the centres of those two nanoparticles, $\widehat{r}_{i j}$ is the unit vector of the direction that connects the $i$ and $j$ nanoparticles, $\widehat{\mu}_{i}$ and $\widehat{\mu}_{j}$ are the unit vectors of the magnetic moments of the $i$ and $j$ nanoparticles, respectively, and $\mu_{j}$ is the magnetic moment of the $j$ nanoparticle.

\section{Phenomenological approach for the dynamics of magnetic relaxation in nanoparticle systems}

We consider the case of aligned magnetic nanoparticle system, randomly distributed in a given volume in an external high frequency alternating magnetic field. We start from the idea of an Ising 2-level model [24] of a spherical nanoparticle system. Some aspects, as the distribution of diameters, effective magnetic anisotropy constants and the three-dimensional geometry can be dealt by numerical simulation [25].

The energy of an $i$ nanoparticle of the system in the $\boldsymbol{H}_{i}$ local magnetic field, oriented along its easy magnetisation axis, is

$$
E_{i}=-\mu_{0} M_{\mathrm{p}, i} H_{i} \cos \theta_{i}-K_{\mathrm{eff}, i} v_{i} \cos ^{2} \theta_{i},
$$

where $v_{i}=\frac{\pi d_{i}^{3}}{6}, \theta_{i}$ being the angle made by the direction of the $i$ nanoparticle magnetic moment $\left(\boldsymbol{M}_{\mathrm{p}, i}\right)$ with the direction of the easy magnetisation axis, along which the external magnetic field is acting. $K_{\mathrm{eff}, i}$ is the effective anisotropy constant of the nanoparticle, and $v_{i}$ is the magnetic core volume of the nanonanoparticle.

Please note that the sizes of nanoparticles and their effective magnetic anisotropy constants may have distributions.
In these conditions, the minimum energy determines the equilibrium properties of the particle, as they correspond to the stable magnetisation directions. The minimum energy (3) must satisfy the two simultaneous conditions

$$
\frac{\partial E_{i}\left(\theta_{i}\right)}{\partial \theta_{i}}=0, \quad \frac{\partial^{2} E_{i}\left(\theta_{i}\right)}{\partial \theta_{i}^{2}}>0 .
$$

A simple mathematical calculation shows that the $\boldsymbol{M}_{\mathrm{p}, i}$ magnetic moment of a given $i$ nanoparticle can be in one of the two equilibrium states, with the minimum energies $E_{\min 1}^{i}$ and $E_{\min 2}^{i}$ determined by $\theta_{i}=0$ and $\theta_{i}=\pi$, separated by the barrier height $E_{\max }^{i}$.

In the presence of thermal fluctuations, the magnetic moment of an $i$ nanoparticle in a stable state, with the minimum energy $E_{\min 1}^{i}$, can spontaneously change its direction to the minimum energy state $E_{\min 2}^{i}$. The energy barriers for these re-orientations are

$$
\begin{aligned}
& E_{\mathrm{b} 12}^{i}=E_{\max }^{i}-E_{\min 1}^{i}, \\
& E_{\mathrm{b} 21}^{i}=E_{\max }^{i}-E_{\min 2}^{i} .
\end{aligned}
$$

We consider that the system is in thermal equilibrium and has $N$ nanoparticles. The average number of nanoparticles passing in time unit from a minimum to another minimum is proportional with $N_{k} \exp \left(-\frac{E_{\mathrm{b} k}}{k_{\mathrm{B}} T}\right)$, where $k_{\mathrm{B}}$ is the Boltzmann constant, and $T$ is the temperature. If $N_{1}$ is the average number of nanoparticles in the state with minimum average energy $\left\langle E_{\min 1}\right\rangle$ and $N_{2}$ is the number of nanoparticles in the minimum average energy $\left\langle E_{\min 2}\right\rangle$, then the equilibrium condition shall be

$$
N_{1} \exp \left(-\frac{\left\langle E_{\mathrm{b} 12}\right\rangle}{k_{\mathrm{B}} T}\right)=N_{2} \exp \left(-\frac{\left\langle E_{\mathrm{b} 21}\right\rangle}{k_{\mathrm{B}} T}\right),
$$

where $\left\langle E_{\mathrm{b} 12}\right\rangle$ and $\left\langle E_{\mathrm{b} 21}\right\rangle$ are the average energy barriers.

Near-equilibrium, the total magnetic moment of the system, in a given moment, is proportional with the difference $N_{1}-N_{2}$. Within an infinitesimal time period, the $n$ difference becomes

$$
\begin{gathered}
\frac{\partial n}{\partial t}=f_{0}\left[N_{1} \exp \left(-\frac{\left\langle E_{\mathrm{b} 12}\right\rangle}{k_{\mathrm{B}} T}\right)\right. \\
\left.-N_{2} \exp \left(-\frac{\left\langle E_{\mathrm{b} 21}\right\rangle}{k_{\mathrm{B}} T}\right)\right],
\end{gathered}
$$

where $f_{0}$ is a factor measured in $\mathrm{s}^{-1}$.

Having in view the relations between $N_{1}, N_{2}$ and $n$, we can write

$$
N_{1}=\frac{N+n}{2}, \text { and } N_{2}=\frac{N-n}{2} .
$$

After applying a simple calculation artifice

$$
\begin{aligned}
& \exp \left(-\frac{\left\langle E_{\mathrm{b} 12}\right\rangle}{k_{\mathrm{B}} T}\right)=\exp \left(-\frac{\left\langle E_{\mathrm{b} 12}\right\rangle-\left\langle E_{\mathrm{b} 21}\right\rangle}{2 k_{\mathrm{B}} T}\right) \\
& \quad \times \exp \left(-\frac{\left\langle E_{\mathrm{b} 12}\right\rangle+\left\langle E_{\mathrm{b} 21}\right\rangle}{2 k_{\mathrm{B}} T}\right), \\
& \exp \left(-\frac{\left\langle E_{\mathrm{b} 21}\right\rangle}{k_{\mathrm{B}} T}\right)=\exp \left(\frac{\left\langle E_{\mathrm{b} 12}\right\rangle-\left\langle E_{\mathrm{b} 21}\right\rangle}{2 k_{\mathrm{B}} T}\right)
\end{aligned}
$$




$$
\times \exp \left(-\frac{\left\langle E_{\mathrm{b} 12}\right\rangle+\left\langle E_{\mathrm{b} 21}\right\rangle}{2 k_{\mathrm{B}} T}\right) .
$$

With the relations (9), (10) and (11), Eq. (8) becomes

$$
\begin{aligned}
\frac{\partial n}{\partial t} & =-f_{0} \exp \left(-\frac{\left\langle E_{\mathrm{b} 12}\right\rangle+\left\langle E_{\mathrm{b} 21}\right\rangle}{2 k_{\mathrm{B}} T}\right) \\
\times & {\left[n \cosh \left(\frac{\left\langle E_{\mathrm{b} 12}\right\rangle-\left\langle E_{\mathrm{b} 21}\right\rangle}{2 k_{\mathrm{B}} T}\right)\right.} \\
& \left.+N \sinh \left(\frac{\left\langle E_{\mathrm{b} 12}\right\rangle-\left\langle E_{\mathrm{b} 21}\right\rangle}{2 k_{\mathrm{B}} T}\right)\right] .
\end{aligned}
$$

Generally, the magnetic relaxation process of the system is governed by such an equation

$$
\begin{aligned}
\frac{\partial M}{\partial t} \cong-f_{0} \exp \left(-\frac{\left\langle E_{\mathrm{b} 12}\right\rangle+\left\langle E_{\mathrm{b} 21}\right\rangle}{2 k_{\mathrm{B}} T}\right) \\
\times\left[M \cosh \left(\frac{\left\langle E_{\mathrm{b} 12}\right\rangle-\left\langle E_{\mathrm{b} 21}\right\rangle}{2 k_{\mathrm{B}} T}\right)\right. \\
\left.+M_{\mathrm{sat}} \sinh \left(\frac{\left\langle E_{\mathrm{b} 12}\right\rangle-\left\langle E_{\mathrm{b} 21}\right\rangle}{2 k_{\mathrm{B}} T}\right)\right],
\end{aligned}
$$

where $M_{\text {sat }}$ is the saturation magnetisation (i.e. the magnetisation of the nanoparticle assembly when all the nanoparticles are aligned along the direction of the external magnetic field), $M$ being the total magnetic moment of the system.

Very close to equilibrium, Eq. (13) becomes

$$
\begin{aligned}
& \frac{\partial M}{\partial t} \cong-2 f_{0} M \exp \left(-\frac{\left\langle E_{\mathrm{b} 12}\right\rangle+\left\langle E_{\mathrm{b} 21}\right\rangle}{2 k_{\mathrm{B}} T}\right) \\
& \quad \times \cosh \left(\frac{\left\langle E_{\mathrm{b} 12}\right\rangle-\left\langle E_{\mathrm{b} 21}\right\rangle}{2 k_{\mathrm{B}} T}\right) .
\end{aligned}
$$

We consider $\left\langle E_{\mathrm{b}}\right\rangle=\frac{\left\langle E_{\mathrm{b} 12}\right\rangle+\left\langle E_{\mathrm{b} 21}\right\rangle}{2}$, which represents the effective energy barrier. With this notation, Eq. (14) will be

$$
\begin{gathered}
\frac{\partial M}{\partial t} \cong-2 f_{0} M \exp \left(-\frac{\left\langle E_{\mathrm{b}}\right\rangle}{k_{\mathrm{B}} T}\right) \\
\times \cosh \left(\frac{\left\langle E_{\mathrm{b} 12}\right\rangle-\left\langle E_{\mathrm{b} 21}\right\rangle}{2 k_{\mathrm{B}} T}\right) .
\end{gathered}
$$

The solution of the differential Eq. (15) gives the evolution equation for the residual magnetisation of the system which, for $H=$ constant during the relaxation, is

$$
\begin{aligned}
& M(t) \cong M(0) \exp \left[-2 t f_{0} M \exp \left(-\frac{\left\langle E_{\mathrm{b}}\right\rangle}{k_{\mathrm{B}} T}\right)\right. \\
& \left.\quad \times \cosh \left(\frac{\left\langle E_{\mathrm{b} 12}\right\rangle-\left\langle E_{\mathrm{b} 21}\right\rangle}{2 k_{\mathrm{B}} T}\right)\right],
\end{aligned}
$$

where $M(0)$ is the system magnetisation at the beginning of the relaxation process.

In the following relation, we use the notation $\tau_{0}=1 / f_{0}$,

$$
\tau_{\mathrm{N}}=\frac{\tau_{0} \exp \left(\frac{\left\langle E_{\mathrm{b}}\right\rangle}{k_{\mathrm{B}} T}\right)}{2 \cosh \left(\frac{\left\langle E_{\mathrm{b} 12}\right\rangle-\left\langle E_{\mathrm{b} 21}\right\rangle}{2 k_{\mathrm{B}} T}\right)},
$$

$\tau_{\mathrm{N}}$ being the Néel relaxation time.

\section{Stochastic approach for the magnetic relaxation dynamics in nanoparticle systems}

The starting point for calculation of the relaxation time is the Fokker-Planck equation [26]. This equation was solved by Brown [27] in case of high and low energy barrier. In the intermediate region, there is no analytical solution. In the Fokker-Planck equation the orientation of magnetization takes continuum values but in practice calculations, in base of real experimental systems, magnetization spends most time in orientations near to the local minima of the energy so it is possible to treat the Fokker-Planck equation in the discrete orientation approximation [28].

The discrete orientation approximation assumes that the magnetic moment rotation due to the thermal fluctuations can be modelled through the equation of probability, for finding the magnetic moments of the particles at the $t$ moment, in the $i$ equilibrium state $(i=1,2)$ [29]. Under the same assumptions and using the same notations as in the previous paragraph, the situation is illustrated in Fig. 1.

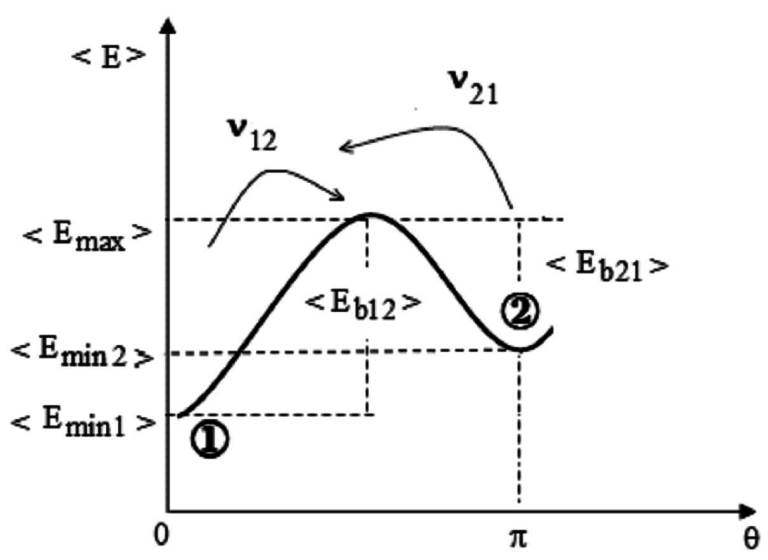

Fig. 1. The average energy state of the nanoparticle system.

Let $P_{1}(t)$ be the probability of finding particles in state 1 at the moment $t$, and $P_{2}(t)$ the probability of finding particles in state 2 at the moment $t$. Let us denote with $\nu_{12}$ the probability of transition, in time unit, from the state 1 into state 2, passing through the maximum, and with $\nu_{21}$ the probability of transition, in time unit, from the state 2 into state 1, passing also through the maximum. With these notations, we obtain $[29,30]$ :

$$
\frac{\mathrm{d} P_{1}}{\mathrm{~d} t}=\nu_{21} P_{2}(t)-\nu_{12} P_{1}(t)
$$

provided that the normalisation condition

$$
\begin{aligned}
& P_{1}(t)+P_{2}(t)=1, \\
& \nu_{i j}=\frac{1}{\tau_{i j}}=\frac{1}{\tau_{0}} \exp \left(-\frac{\left\langle E_{\mathrm{b} i j}\right\rangle}{k_{\mathrm{B}} T}\right),
\end{aligned}
$$

with $f_{0}=\tau_{0}^{-1}=$ attempt frequency (which can be experimentally determined for each system). 
At thermal equilibrium [30]:

$$
\frac{\nu_{21}^{\mathrm{T}}}{\nu_{12}^{\mathrm{T}}}=\exp \left(-\frac{\varepsilon}{k_{\mathrm{B}} T}\right) \text {, with } \varepsilon=\left\langle E_{\min 1}\right\rangle-\left\langle E_{\min 2}\right\rangle \text {. }
$$

Considering the normalisation condition (19), the solution of Eq. (18) is

$$
P_{1}(t)=\frac{\nu_{21}^{\mathrm{T}}}{\nu^{\mathrm{T}}}-\frac{\nu_{12}^{\mathrm{T}}}{\nu^{\mathrm{T}}} \exp \left(-t \nu^{\mathrm{T}}\right)
$$

with

$$
\nu^{\mathrm{T}}=\nu_{12}^{\mathrm{T}}+\nu_{21}^{\mathrm{T}} .
$$

By inserting the relations (20) and (21) into (22), we obtain

$$
\begin{aligned}
& P_{1}(t)=\frac{1-\exp \left(\frac{\varepsilon}{k_{\mathrm{B}} T}\right) \exp \left(-\frac{t}{\tau_{\mathrm{N}}}\right)}{1+\exp \left(\frac{\varepsilon}{k_{\mathrm{B}} T}\right)}, \\
& P_{2}(t)=\frac{\exp \left(\frac{\varepsilon}{k_{\mathrm{B}} T}\right)\left[1+\exp \left(-\frac{t}{\tau_{\mathrm{N}}}\right)\right]}{1+\exp \left(\frac{\varepsilon}{k_{\mathrm{B}} T}\right)} .
\end{aligned}
$$

In Eqs. (24) and (25), $\tau_{\mathrm{N}}$ has the significance of average Néel relaxation time

$$
\begin{gathered}
\tau_{\mathrm{N}}^{-1}=\nu^{\mathrm{T}}=\sum_{i \neq j} \frac{1}{\tau_{i j}}=\frac{1}{\tau_{12}}+\frac{1}{\tau_{21}}=\nu_{12}^{\mathrm{T}}+\nu_{21}^{\mathrm{T}}= \\
\nu_{21}^{\mathrm{T}} \exp \left(\frac{\varepsilon}{k_{\mathrm{B}} T}\right)+\nu_{21}^{\mathrm{T}}=\nu_{21}^{\mathrm{T}}\left[1+\exp \left(\frac{\varepsilon}{k_{\mathrm{B}} T}\right)\right]= \\
\frac{1}{\tau_{0}} \exp \left(-\frac{\left\langle E_{\mathrm{b} 21}\right\rangle}{k_{\mathrm{B}} T}\right)\left[1+\exp \left(\frac{\varepsilon}{k_{\mathrm{B}} T}\right)\right]
\end{gathered}
$$

or, by using the second equation:

$$
\begin{aligned}
& \tau_{\mathrm{N}}^{-1}=\frac{1}{\tau_{12}}+\frac{1}{\tau_{21}}=\frac{1}{\tau_{0}}\left[\exp \left(-\frac{\left\langle E_{\mathrm{b} 21}\right\rangle}{k_{\mathrm{B}} T}\right)\right. \\
& \left.\quad+\exp \left(-\frac{\left\langle E_{\mathrm{b} 12}\right\rangle}{k_{\mathrm{B}} T}\right)\right] .
\end{aligned}
$$

Taking into account Eqs. (10) and (11), Eq. (27) can be brought to the form

$$
\begin{gathered}
\tau_{\mathrm{N}}^{-1}=\frac{1}{\tau_{0}} \exp \left(-\frac{\left\langle E_{\mathrm{b} 12}\right\rangle+\left\langle E_{\mathrm{b} 21}\right\rangle}{2 k_{\mathrm{B}} T}\right) \\
\times\left[\exp \left(\frac{\left\langle E_{\mathrm{b} 12}\right\rangle-\left\langle E_{\mathrm{b} 21}\right\rangle}{2 k_{\mathrm{B}} T}\right)+\exp \left(-\frac{\left\langle E_{\mathrm{b} 12}\right\rangle-\left\langle E_{\mathrm{b} 21}\right\rangle}{2 k_{\mathrm{B}} T}\right)\right] .
\end{gathered}
$$

Having in view that $\exp (x)+\exp (-x)=2 \cosh (x)$, we obtain

$$
\begin{aligned}
\frac{1}{\tau_{\mathrm{N}}} & =\frac{1}{\tau_{0}} 2 \exp \left(-\frac{\left\langle E_{\mathrm{b} 12}\right\rangle+\left\langle E_{\mathrm{b} 21}\right\rangle}{2 k_{\mathrm{B}} T}\right) \\
& \times \cosh \left(\frac{\left\langle E_{\mathrm{b} 12}\right\rangle-\left\langle E_{\mathrm{b} 21}\right\rangle}{2 k_{\mathrm{B}} T}\right), \\
\tau_{\mathrm{N}} & =\frac{\tau_{0} \exp \left(\frac{\left\langle E_{\mathrm{b}}\right\rangle}{k_{\mathrm{B}} T}\right)}{2 \cosh \left(\frac{\left\langle E_{\mathrm{b} 12}\right\rangle-\left\langle E_{\mathrm{b} 21}\right\rangle}{2 k_{\mathrm{B}} T}\right)} .
\end{aligned}
$$

If we compare the result obtained through the stochastic approach — relation (30) — with the result obtained when using the phenomenological model - relation (16), we can easily see that they are practically identical. Both approximations treat the case close to equilibrium.

\section{Results and discussions}

In this paper, we discussed about two approaches (phenomenological and stochastic) regarding the Néel relaxation time, approaches used in the multitude of simulation models found in literature, approaches that have led to the same result. These approaches are appropriate for aligned magnetic nanoparticle systems in alternative magnetic fields (as in case of ultra-high density magnetic data storage devices), although they are frequently used in the literature also in the case when the magnetic anisotropy axes of the nanoparticles have a random orientation.

Given that the measurements carried out experimentally on a nanoparticle magnetic system are very difficult to interpret, because of overlapping of various effects that cannot be separated one from each other, the development of more reliable models to study these systems provides the understanding of phenomena at nanoscopic level, which is a vital understanding for obtaining a certain control of the system behaviour in the various applications.

To show how these approaches can be integrated into a model, we consider a magnetite nanoparticle system aligned in a solid non-magnetic matrix, under the action of an external alternating magnetic field with high frequency and given amplitude, applied in the direction of the aligned anisotropy axes. The nanoparticle positions are random in a given volume, according to the nanoparticle volume fraction. We considered spherical uncoated magnetite nanoparticles having the density of $5180 \mathrm{~kg} / \mathrm{m}^{3}$ [31], saturation magnetisation $M_{\mathrm{s}}=4.46 \times 10^{5} \mathrm{~A} / \mathrm{m}$ [31], and uniaxial magnetic anisotropy with the average effective magnetic anisotropy constant $K_{\text {eff }}=25 \times 10^{3} \mathrm{~J} / \mathrm{m}^{3}$ [31]. The analysed systems contained nanoparticles having the volume fraction $f=0.1$ and average diameters $=10 \mathrm{~nm}$ at the temperature $T=293 \mathrm{~K}$. The external magnetic field taken into account had the amplitude of $150 \mathrm{kA} / \mathrm{m}$. The local magnetic field acting on the $i$-th nanoparticle was calculated using direct summation. In many papers, the attempt frequency is considered to be $f_{0}=10^{9} \mathrm{~s}^{-1}$ [32], or to fall within various ranges, such as $\left(10^{13}-10^{9}\right) \mathrm{s}^{-1}$ [33], and to depend only on the material properties. We considered that the nanoparticle sizes and effective magnetic anisotropy constants had a lognormal distribution, the standard shape deviations were $v_{\mathrm{d}} \mathrm{d}_{\mathrm{m}}$ and $v_{\text {Keff }} K_{\text {eff,m }}$, i.e. $v_{\mathrm{d}}$ and $v_{\text {Keff }}$ are standard deviation parameters, $d_{\mathrm{m}}$ the average particle diameter and $K_{\mathrm{eff}, \mathrm{m}}$ - their effective magnetic anisotropy constant.

The extended theoretical predictions developed in the previous sections led to the same final result, Eq. (30), which is the basis of our simulation. For each nanoparticle, we calculate the energy barriers $E_{\mathrm{b} 12}^{i}$ and $E_{\mathrm{b} 21}^{i}$. Figure 2 shows the histograms of energy barriers for a system consisting of 5000 nanoparticles, having the above mentioned properties, with distribution of effective magnetic anisotropy constants $\left(v_{\text {Keff }}=0.1\right)$ and size distribution $\left(v_{\mathrm{d}}=0.1\right)$. 


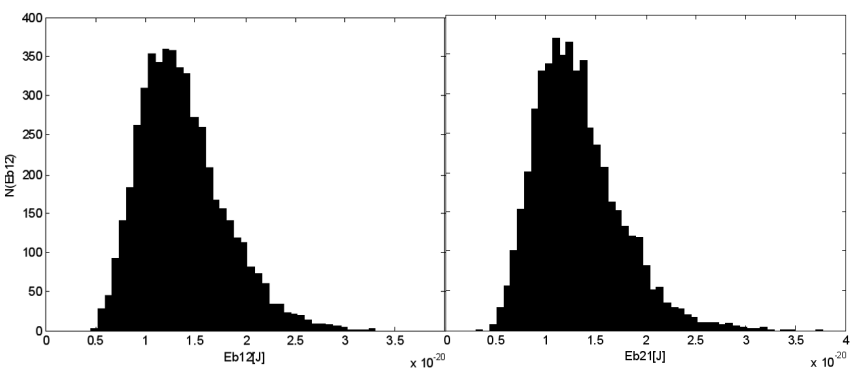

Fig. 2. Histograms of energy barriers for a system consisting of 5000 magnetite nanoparticles, with distribution of sizes and effective magnetic anisotropy constants.

Then, we calculate the average energy barriers $\left\langle E_{\mathrm{b} 12}\right\rangle$, $\left\langle E_{\mathrm{b} 21}\right\rangle$ and $\left\langle E_{\mathrm{b}}\right\rangle$. In the examined case, $\left\langle E_{\mathrm{b} 12}\right\rangle=$ $1.3824 \times 10^{-20} \mathrm{~J},\left\langle E_{\mathrm{b} 21}\right\rangle=1.3217 \times 10^{-20} \mathrm{~J}$, and $\left\langle E_{\mathrm{b}}\right\rangle=1.35205 \times 10^{-20} \mathrm{~J}$. Then, based on the relation (30), we calculate the Néel relaxation time. In this case, $t_{\mathrm{N}}=3.3065 \times 10^{-9} \mathrm{~s}$.

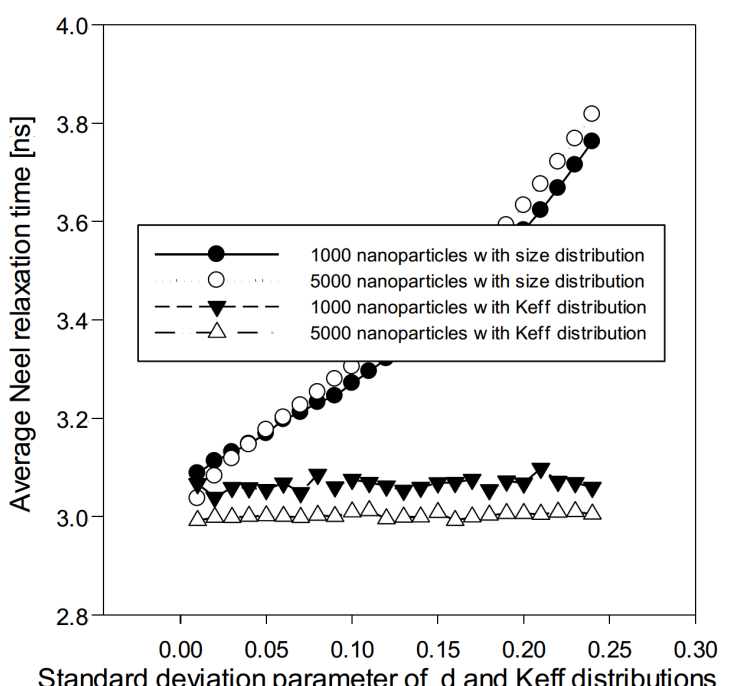

Fig. 3. Average Néel relaxation time versus standard deviation parameter of the distributions.

Figure 3 shows the average Néel relaxation time versus the standard deviation parameter of size and effective magnetic anisotropy constant distributions. When studying the contribution of size distribution, we maintain the value of the effective magnetic anisotropy constant, and vice versa. We can see that the size distribution is strongly influenced by the Néel relaxation time. The average Néel relaxation time increases slightly with increasing standard deviation parameter of the effective magnetic anisotropy constant distribution. In this way, one can distinctively study the influence of physical parameters affecting the magnetic relaxation.

Figure 4 shows the average Néel relaxation time versus the volume fraction of nanoparticles for the system consisting of 5000 magnetite nanoparticles, firstly examined in terms of size distribution, then in terms of effective

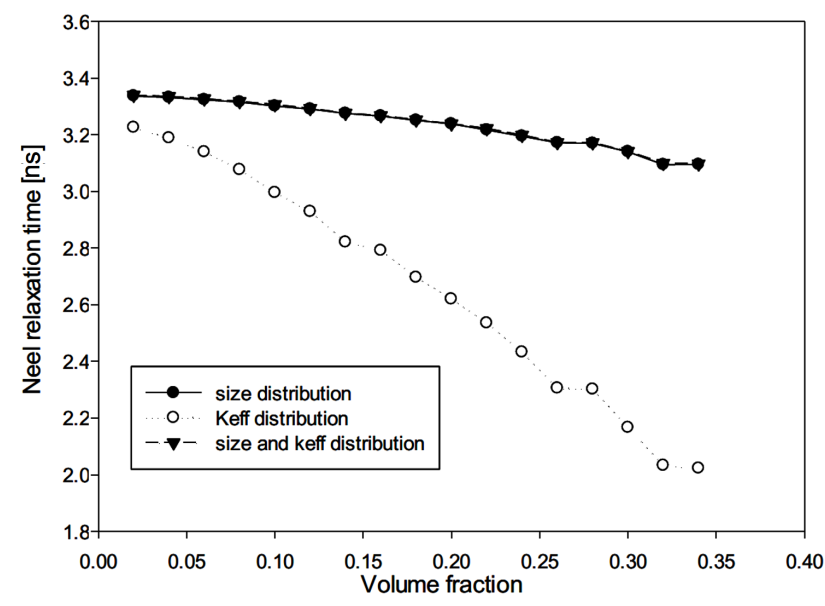

Fig. 4. Néel relaxation time versus the volume fraction of nanoparticles.

magnetic anisotropy constant distribution, and then by taking into account the case when the effective magnetic anisotropy constant distribution is superimposed over the size distribution. In all three cases, it can be noticed a decrease in Néel relaxation time with increasing volume fraction of nanoparticles. This behaviour is confirmed by the scientific literature, in theoretical as well as experimental works $[34,35]$. This happens because the local magnetic field increases and the energy barriers decrease with increasing concentration.

\section{Conclusions}

In this paper, by two different routes with respect to the dynamics of the magnetic moments of a system of magnetic nanoparticles with magnetic dipole interactions, a phenomenological route and a stochastic one, we obtained the same result, i.e. the same relation expressing the average Néel relaxation time related to the system. Therefore, there are two methods to investigate the dynamics of the magnetic moments in aligned magnetic nanoparticle systems with magnetic dipole interactions, methods that lead to the same results, which are important to highlight. The paper also shows a modality to use these approaches for creating a model to simulate the average Néel relaxation time for aligned magnetic nanoparticle systems, and a modality to study the influence of various physical parameters that characterise the dynamic behaviour of the system.

\section{References}

[1] K.M. Krishnan, IEEE Trans. Magn. 46, 2523 (2010).

[2] Q.A. Pankhurst, J. Connolly, S.K. Jones, J. Dobson, J. Phys. D Appl. Phys. 36, R167 (2003).

[3] Magnetic Cell Separation, Eds. M. Zborowski, J. Chalmers, Vol. 32, Laboratory Techniques in Biochemistry and Molecular Biology, Elsevier, Amsterdam 2008. 
[4] H.B. Na, I.C. Song, T. Hyeon, Adv. Mater. 21, 2133 (2009).

[5] K. Hamad-Schifferli, J.J. Schwartz, A.T. Santos, S. Zhang, J.M. Jacobson, Nature 415, 152 (2002).

[6] S.C. McBain, U. Griesenbach, S. Xenariou, A. Keramane, C.D. Batich, E.W.F. Falton, J. Dobson, Nanotechnology 19, 405102 (2008).

[7] S. Takeda, B. Terazono, F. Mishima, H. Nakagami, S. Nishijima, Y. Kaneda, J. Nanosci. Nanotechnol. 6, 3269 (2006).

[8] F.J. Boehm, C. Aicheng, Recent Patents Biomed. Eng. 2, 30 (2009).

[9] Y. Yuan, D.A. Tasciuc Borca, J. Magn. Magn. Mater. 323, 2463 (2011).

[10] V. Sreeja, P. Joy, Int. J. Nanotechnol. 8, 907 (2011).

[11] T. Neuberger, B. Schopf, H. Hofmann, M. Hofmann, B. von Rechenberg, J. Magn. Magn. Mater. 293 , 483 (2005).

[12] S.M. Bird, J.M. Galloway, A.E. Rawlings, J.P. Bramble, S.S. Staniland, Nanoscale 7, 7340 (2015).

[13] L. Néel, C.R. Acad. Sci. Paris 228, 664 (1949).

[14] W.F. Brown Jr., Magnetostatic Principles in Ferromagnetism, North-Holland, Amsterdam 1962.

[15] S. Shtrikman, E.P. Wohlfarth, Phys. Lett. A 85, 457 (1981).

[16] J.L. Dormann, L. Bessais, D. Fiorani, J. Phys. C 21 , 2015 (1988).

[17] M.F. Hansen, S. Mørup, J. Magn. Magn. Mater. 184, 262 (1998).

[18] Tran Nguyen Lan, Tran Hoang Hai, Comput. Mater. Sci. 49, S287 (2010).

[19] R. Sharma, C. Pratima, S. Lamba, S. Annapoorni, Pramana J. Phys. Indian Acad. Sci. 65, 739 (2005).

[20] S. Ruta, O. Hovorka, R. Chantrell, Sci. Rep. 5, 9090 (2015).
[21] D.B. Reeves, J.B. Weaver, Appl. Phys. Lett. 104, 102403 (2014).

[22] P.M. Déjardin, J. Appl. Phys. 110, 113921 (2011).

[23] C. Haase, U. Nowak, Phys. Rev. B 85, 045435 (2012).

[24] R. Prozorov, Y. Yeshurun, Phys. Rev. B 59, 6959 (1999).

[25] M. Osaci, M. Panoiu, T. Heput, I. Muscalagiu, Appl. Math. Model. 30, 545 (2006).

[26] W.T. Coffey, P.J. Cregg, Y. U. P. Kalmykov On the Theory of Debye and Néel Relaxation of Single Domain Ferromagnetic Particles, Advances in Chemical Physics, Vol. LXXXIII, Wiley, New York 1993.

[27] W.F. Brown Jr., Phys. Rev. 130, 1677 (1963).

[28] W.T. Coffey, Yu.P. Kalmykov, The Langevin Equation: With Applications to Stochastic Problems in Physics, Chemistry and Electrical Engineering, World Scientific Series in Contemporary Chemical Physics, Vol. 27, World Sci. Publ., London 2012.

[29] L. Lopez-Diaz, L. Torres, E. Moro, Phys. Rev. B 65 , 224406 (2002).

[30] F. Reif, Fundamentals of Statistical and Thermal Physics, McGraw-Hill, New York 1965.

[31] R.E. Rosensweig, J. Magn. Magn. Mater. 252, 370 (2002).

[32] D.L. Beke, Cryst. Res. Technol. 33, 1039 (1998).

[33] S. Mørup, M.F. Hansen, C. Frandsen, Beilstein J. Nanotechnol. 1, 182 (2010).

[34] A. Silva, T. Oliveira, J.B. Mamani, S.M.F. Malheiros, L. Malavolta, L.F. Pavon, T.T. Sibov, E. Amaro Jr., A. Tannús, E.L.G. Vidoto, M.J. Matins, R.S. Santos, L.F. Gamarra, Int. J. Nanomed. 6, 591 (2011).

[35] K. Parvin, J. Ma, J. Ly, X.C. Sun, D.E. Nikles, K. Sun, L.M. Wang, J. Appl. Phys. 95, 7121 (2004). 